\title{
The estimation of risks from the induction of recessive mutations after exposure to ionising radiation
}

\author{
A G SEARLE* AND J H EDWARDS $\dagger$ \\ From * the Medical Research Council Radiobiology Unit, Chilton, Didcot, Oxon OX11 ORD; and the Genetics \\ Laboratory, Department of Biochemistry, University of Oxford, South Parks Road, Oxford OXI 3QU.
}

SUMMARY Since recent assessments of genetic risks from radiation have concentrated on harmful dominant effects, a quantitative assessment of risks from recessives is needed. Presumably, harmful recessives can arise at all loci coding for essential proteins (perhaps 10 000), but mutation to dominant alleles is likely to be a property of relatively few loci. While many recessives doubtless remain to be discovered, those known at present tend to have earlier and more severe effects than dominants. Induced recessive mutations can cause harm by (1) partnership with a defective allele already established in the population; (2) partnership with another recessive mutation induced at the same locus; (3) the formation of homozygous descendants, that is, identity by descent; and (4) heterozygous effects. Calculations based on a combination of data from observations on human populations and from mouse experiments suggest that an extra genetically significant dose of $1 \mathrm{cGy}$ (centiGray, equivalent to $1 \mathrm{rad}$ ) $\mathrm{X}$ or $\gamma$ irradiation received by each parent in a stable population with a million liveborn offspring would induce up to 1200 extra recessive mutations. From partnership effects, about one extra case of recessive disease would be expected in the following 10 generations. Homozygosity resulting from identity by descent could not normally occur until the fourth generation after exposure but, on certain assumptions, about ten extra cases of recessive disease would be expected from this cause by the tenth generation. In the same period, about 250 recessive alleles would be eliminated in heterozygotes (that is, Muller's 'genetic deaths') given $2 \cdot 5 \%$ heterozygous disadvantage. These deleterious heterozygous effects should not be combined with those of dominants, as has been done in some previous risk estimates. It is considered unlikely that many radiation induced recessives would show heterozygous advantage. Certain dominants (combined frequency at least $10^{-3}$ ) should be excluded from calculations of mutational risk because they are unlikely to be maintained by mutation.

In recent years, national and international committees have made detailed estimates of the magnitude of genetic risks from radiation. With respect to mutational diseases, however, the detail has extended only to risks from dominant and $\mathrm{X}$ linked conditions. In the 1972 UNSCEAR Report, ${ }^{2}$ only the first generation risk from the adverse effects of recessives in heterozygous condition was given, based on data from Drosophila. However, in the 1972 BEIR $^{3}$ report and in subsequent BEIR ${ }^{4}$ and UNSCEAR $^{5}{ }^{6}$ reports, as well as in the report of an ICRP Task Group, ${ }^{7}$ risks from heterozygous effects

Received for publication 16 August 1985.

Accepted for publication 19 September 1985. of recessives have been regarded as one component of the risks from dominant disorders, while the risks of homozygous recessive disorders have been described as "relatively slight" or "very few" in the first generation with a "very slow increase" to an equilibrium frequency. Thus, none of the committees reporting in the past 12 years has attempted to give numerical estimates of recessive risk, while all have followed almost exactly the vague wording adopted by the 1972 BEIR report, ${ }^{3}$ without attempting to justify this by calculations. This contrasts with the numerical estimates given for the extra risks of dominant and $\mathrm{X}$ linked diseases.

The present paper attempts to correct this imba- 
lance and thus to allow a more precise assessment of the relative risks of inducing dominant and recessive disorders after additional radiation exposures. It should be noted that these exposures are assumed to be genetically significant, that is, received by the gonads before offspring are conceived.

\section{A comparison of dominant and recessive diseases}

UNSCEAR ${ }^{8}$ considered that clinically recognised autosomal recessive disorders were, on average, much more severe in their effects than dominant disorders. ${ }^{9}$ However, a comprehensive comparison of all dominants and recessives is very difficult for the following reasons. Severe dominants which kill early or impair reproduction are non-heritable and therefore unrecognisable as dominant by pedigree studies, while recessives, however severe, are transmitted in the heterozygous condition and can therefore be recognised, in theory at least, as recessives through recurrence in sibships and sometimes through association with consanguinity. If, however, they lead to prenatal death (like many induced recessives in the mouse) they will usually be undetected in man against a background of at least $15 \%$ of failed conceptions.

It seems reasonable to expect a heavier genetic load from recessives than from dominants because (1) only a limited number of loci are likely to be capable of mutation to harmful dominant alleles, because this requires that the well buffered action of the wild type allele is overridden, ${ }^{9 a}$ while (2) probably all loci coding for essential proteins, perhaps of the order of 10000 , can give recessive alleles. Fewer than 1000 of these recessives have been recognised so far, so new recessive mutants in isolated human populations are likely to be really new and impossible to recognise for several generations. ${ }^{10}$ McKusick ${ }^{11}$ lists 934 confirmed dominants as against 588 confirmed recessives, or almost 3:2. This contrasts with a figure (calculated from Peters ${ }^{12}$ ) of $33 \%$ known dominants, or $1: 2$, in the laboratory mouse (if codominants at biochemical and immunological loci are excluded), in which the existence of many inbred strains facilitates detection of recessives.

Most recessive disorders show up in infancy or childhood, while many of the commoner recognised dominants first appear in adult life, when they may be difficult to distinguish from the common, but poorly understood, disabling effects of age, including blindness (especially from cataraci), deafness, and dementia. Some severe dominants, including Huntington's chorea and myotonic dystrophy, so rarely appear as new mutants in well conducted surveys $^{1:-15}$ that their population frequency may well be maintained by some sort of heterozygous advantage during the reproductive period rather than by mutation. The dominant hyperlipidaemias and hypercholesterolaemias are also so common that they are unlikely to be maintained by mutation. These should therefore be excluded from calculations of mutational risk. The combined frequency of the former two disorders in the UK is about 0.7 per 1000 livebirths, ${ }^{16}$ while the latter two are considerably commoner. Similarly, there are recessive disorders (such as the thalassaemias, sickle cell disease, and cystic fibrosis) which are so common in some racial groups and so rare in others that heterozygous advantage, either now or in the past, must be postulated; this has been demonstrated in sickle cell disease.

\section{Sources of homozygous recessive effect}

Unlike the situation with dominant mutations, three distinct sources of effect need to be considered in assessing the amount of additional genetic disease which would arise from the induction of recessive mutations, in addition to possible heterozygous effects. The first source is the coming together of a new mutation with a mutant allele at the same locus already present in the population. The second is the coming together of two recessive mutations independently induced at the same locus. The third is through the formation of a homozygous descendant, that is, through identity of descent.

The first and second categories of effect might occur in any generation after exposure. However, the second would require the chance combination of two very rare events and can be disregarded at the low levels of radiation exposure which are considered here. Since the third category depends on the attainment of homozygosity by a newly induced recessive it can, ignoring incest, only happen after the third generation, or about a century later.

\section{Experimental evidence}

Before attempting to estimate the mutagenic risks from these sources, it is necessary to consider relevant data from the extensive series of experiments in the mouse on the induction of recessive mutations by ionising radiation. A number of these mutations are known to be small deficiencies which also include neighbouring loci. ${ }^{17}$

Most information is from experiments which detect recessives at a specific set of loci. At the $6 \mathrm{~Gy}$ level of spermatogonial $X$ irradiation, available data $^{18-20}$ give an overall frequency of $135 \times 10^{-6}$ per locus for the original set of seven specific loci. A new set of six loci, however, gave a frequency of $47 \times 10^{-6}$ per locus at the same dose level, ${ }^{21}$ which 
suggested that the original set of seven loci might be more mutable than average. A recent specific locus test at a different dose level and with another set of loci has tended to confirm this idea. ${ }^{22}$ An average mutation frequency of $94 \times 10^{-6}$ per locus is obtained by combining results at the $6 \mathrm{~Gy}$ level, that is, a rate of $16 \times 10^{-6}$ per locus per Gy, assuming linearity. Since the ratio of induced mutation rates after chronic and acute exposures is $0 \cdot 33,{ }^{2.3}$ the expected mutation rate to recessives after chronic spermatogonial exposure would be about $5 \cdot 2 \times 10^{-6}$ per locus per Gy. Although there is evidence that induced specific locus mutation rates in mouse oocytes after chronic exposures are decidedly lower than in spermatogonia, ${ }^{24}$ the situation in man is less clear, so that it might be advisable to assume a similar figure in both sexes.

Of 206 recessive mutations induced at the original set of seven specific loci by spermatogonial irradiation, 136 were lethal in the homozygote, ${ }^{25}$ but effects varied greatly between loci. Many homozygotes died before birth while others were lethal at birth or weaning. However, only four out of 14 recessives were homozygous lethals after similar experiments with the Harwell six locus stock. ${ }^{21}$ Perhaps it would be best to assume that about two-thirds of radiation induced recessives have lethal or deleterious effects in the homozygotes, while in about half of these such effects are neonatal or postnatal. If so, the expected mutation rate of $5.2 \times 10^{-6}$ given in the previous paragraph can be changed to $3.5 \times 10^{-6}$ for mutations to all lethal and deleterious recessives, and to $1.7 \times 10^{-6}$ for mutations to lethal and deleterious recessives which act at or after birth.

These are per locus rates, but attempts have also been made to obtain overall rates of induction of recessive mutations for the whole genome. Analysis of such data from spermatogonial irradiation ${ }^{26}$ gave an overall mutation rate of $0.09 \times 10^{-3} / \mathrm{genome} / \mathrm{rad}$ for recessive lethals acting in utero after acute $X$ irradiation, equivalent to $9 \times 10^{-3} /$ genome/Gy. If (as before) a factor of 0.33 is assumed for the conversion of effects of acute to chronic exposure, and if prenatal lethals constitute half of all harmful recessives, then a figure of $3 \times 10^{-3} /$ genome/ $\mathrm{Gy}$ is obtained for the induction of recessives with lethal or deleterious effects at or after birth.

\section{Partnership of induced and pre-existing recessives}

Experience with the mouse suggests that the usual zygotic consequence of two gametes with recessive alleles, or deficiencies, at the same locus is a phenotype that resembles the homozygote for the less severe allele, or is intermediate between the two homozygotes. Therefore, partnership between an induced prenatal lethal and a previously existing recessive for a serious hereditary disease is likely to lead to a recessive disorder at least as severe as the regular form.

If $\mu$ is the rate of induction of recessive mutations at locus $a$ per cGy of parental irradiation at low doses and dose rates, $q$ the recessive gene frequency at this locus, and $\mathrm{N}$ the total number of offspring from the exposed population, then the number expected to show a recessive phenotype because of interaction of induced and pre-existing recessives at the same loci will be $N \Sigma \mu_{i t} q_{a}$ per cGy.

Estimates of the gene frequency $(\Sigma q)$ of human deleterious recessives may be made from the phenotypic frequencies or, indirectly, from the results of inbreeding. Indirect estimates based on cousin marriage have a very wide range. ${ }^{27}$ If the concept of a recessive is extended to include lethal equivalents, ${ }^{28}$ some four to five equivalents per gamete may be inferred. The rarity of recessive disease in most cousin marriages suggests a low figure. (The incidence in cousin marriage is $n / 16$, where $\mathrm{n}$ is the number of recessives per gamete when $n$ is small or, more exactly, $\left.1-(15 / 16)^{n}\right)$. It is possible that many recessives cause early fetal death. If so this would not be recognised in man, as the known miscarriage rate is about $15 \%$ and, due to the recurrence risk being only $25 \%$, would not be a major cause of infertility. Indeed, such disorders may have been advantageous in spacing births and may be very common in man.

While data on phenotypic frequencies are limited, the frequency of the commonest form in any community is usually known, and the relative frequency of the lesser known forms can be derived with reasonable precision, that is, within a factor of two, from hospital and laboratory sources.

In the UK, excluding immigrants, the incidences of fibrocystic disease and phenylketonuria are well known, at about 1 in 2000 and 1 in 15000 respectively, both varying by area. Congenital adrenal hyperplasia (21-hydroxylase deficiency) has an intermediate incidence. Excluding recessive disorders which lead to blindness or partial sight, deafness, or severe mental handicap (an incidence of about 1,1 , and 3 per 1000 respectively), the number of other recessive disorders diagnosed in hospitals in the UK hardly exceeds the number diagnosed with cystic fibrosis. It seems that in most ethnic groups one or two disorders account for over half the children with recessive disease. Excluding haemoglobinopathies, which are virtually unknown in Northern Europeans, the total incidence of recessive diseases is probably 0.5 to $1 \%$, although only about a tenth of these, or 1 in 1000 , are 
diagnosed within a month of birth. Recessive disorders are still being discovered, but it seems unlikely that the cumulative frequency of severe postnatal recessive disorders as yet unknown would exceed 1 per 1000 .

The gene frequency of a recessive is the square root of the incidence of the homozygote. Therefore, if the square roots of the incidences of the various diseases are summed, this gives the mean number of recessives per gamete $(\Sigma \mathrm{q})$, and is about $1 .^{29}$

The value of $\mu$ (rate of induction of recessives) can only be inferred in man from the experimental data already referred to or from a combination of human and experimental data. Indirect estimates of the natural mutation frequency for human recessives with drastic effects are consistent with a figure of $6 \times 10^{-6} /$ locus/generation, or $21 \times 10^{-6}$ if $\mathrm{de}-$ trimentals are included. ${ }^{30}$ Morton ${ }^{30}$ suggested a doubling dose of $42 \mathrm{rad}$ (about $0.4 \mathrm{~Gy}$ ) for acute irradiation of spermatogonia and oocytes. A higher doubling dose (that is, reduced effectiveness) would be expected for low LET (X or $\gamma$ ) irradiation at low doses or dose rates and the value of $1 \mathrm{~Gy}$ (100 rad) has been used by UNSCEAR. ${ }^{56}$ Schull et al ${ }^{31}$ estimated a mean doubling dose of 156 rems (468 rems for chronic exposures) from data on three indicators of genetic effects in progeny of survivors of the atomic bombings of Hiroshima and Nagasaki, but confidence limits were very wide, ranging from $18 \mathrm{rems}(0 \cdot 18 \mathrm{~Sv})$ to infinity. The average rate of induction of 'drastic' human recessives by chronic exposures to radiation of low LET would be $6 \times 10^{-6} /$ locus/Gy if the doubling dose estimate of $1 \mathrm{~Gy}$ is used.

If the estimate obtained above for $\Sigma q$ of one severely detrimental recessive per human gamete is combined with the average mutation rates of either $3.5 \times 10^{-6}$ (mouse data on all lethal and deleterious recessives) or $6 \times 10^{-6}$ (human data) recessives per locus per Gy of chronic irradiation, then after a genetically significant dose of $1 \mathrm{~Gy}$ to both parents, one would expect about $10(1 \times 3.5 \times 2$ or $1 \times 6 \times 2$ ) extra cases of recessive disease in the offspring per million livebirths from partnership effects. A more realistic exposure level for man made radiation would be $1 \mathrm{cGy}$ ( $1 \mathrm{rad})$, which would lead to $0 \cdot 1$ extra cases per million in the first generation. Figures for the following generations would depend on the extent of heterozygous disadvantage in the induced recessives. Lüning ${ }^{32}$ found little evidence for dominant deleterious effects of induced recessive lethals in mice, although most induced deleterious dominants in mice are lethal in the homozygote. ${ }^{25}$ Crow $^{33}$ concluded, by analogy with Drosophila, that for human recessives "almost all have some deleterious effects in heterozygotes".
Radiation induced recessives, since they seem frequently to involve deficiencies, are likely to be more detrimental in the heterozygote than naturally occurring recessives. In the absence of any reliable estimate of the magnitude of this heterozygous selective disadvantage in man, it seems reasonable to use the figure of $2.5 \%$ which has been obtained from studies of recessive lethals in populations of Drosophila. If so, the cumulative effects per million over 10 generations of a single exposure of 1 cGy can be estimated by calculating the sum over 10 generations of the geometric series $0 \cdot 1$ $\left(1+0.975+0.975^{2} \ldots .0 \cdot 975^{9}\right)$. This equals $0 \cdot 86$, or approximately one extra severely detrimental recessive condition. In the same way the corresponding figure for 100 generations is $3 \cdot 7$, or about 4 . If there were a persistent increase of $1 \mathrm{cGy}$ per generation in chronic exposures to parents, then the number of extra cases of recessive disease would very gradually approach a maximum of about four per million livebirths per generation, being the sum to infinity of the geometric series given above, as the result of this interaction between induced and preexisting recessives.

If induced recessives were at no heterozygous disadvantage, then the cumulative effects would again add up to one extra recessive disorder in 10 generations, but would be about 10 over 100 generations. If the extra exposure continued in each generation, there would be a continued increase in the frequency of extra recessive conditions, without any equilibrium being reached.

\section{Homozygosity of induced recessives}

For this source of recessive risk we are only concerned with induced recessive mutations in which the homozygotes have deleterious effects at or after birth. If there are 10000 loci per genome capable of mutation to such harmful recessives, as judged by our estimate of 10000 essential proteins, then the estimates already given provide the following per genome mutation rates: (1) $17 \times 10^{-3}$ / genome/Gy from the specific locus results; (2) $3 \times 10^{-3} /$ genome/Gy from recessive lethal results; (3) $60 \times 10^{-3} /$ genome/Gy from combined human and mouse (doubling dose) estimates. These estimates vary by a factor of 20 . The last and most pessimistic estimate would mean that if parents in a stable population with $10^{6}$ live born offspring were each exposed to an extra $1 \mathrm{cGy}$ low LET irradiation, then $6 \times 10^{-4} \times 2 \times 10^{6}=1200$ progeny would carry induced recessives of the type indicated.

If we make the assumption, as before, that these newly arisen heterozygotes are at a selective disadvantage of $2 \cdot 5 \%$, then after two more generations 
the above figure of 1200 would have fallen to 1140 $\left(1200 \times 0.975^{2}\right)$ heterozygotes for induced recessives. By the tenth generation it would have fallen to 955; thus, nearly 250 recessive alleles would have been eliminated in heterozygotes. The first homozygotes might be expected to appear in the fourth generation, from first cousin matings. If there were $1 \%$ of these and equal numbers of each sex (that is, 570) who were heterozygous for these recessives, then about six male and about six female heterozygotes would mate with cousins. Since the probability of the cousin also carrying the induced recessive is $25 \%$, three matings between heterozygotes would be expected. As the probability of homozygosity for the recessive in the offspring of such matings is also $25 \%$, then, with two offspring per mating, 1.5 homozygotes would be expected $(3 \times 2 \times 0.25)$ in the fourth generation. With similar calculations, assuming $2.5 \%$ selective disadvantage of recessives per generation, the total number of homozygotes for induced recessives in the first 10 generations after exposure would be about 10 and in the first 100 would be about 50 . If there were no heterozygous disadvantage, these figures would be about 10 and 150 respectively. If the population were exposed to 1 cGy extra radiation per parent in each generation then the number of extra heterozygotes (given $2.5 \%$ disadvantage) would gradually approach a maximum of about 50000 , that is, the sum of $\left(1+0.975+0.975^{2} \ldots\right)$, from which, on the same calculations, about 60 homozygotes per generation would be expected if there were $1 \%$ inbreeding.

It should be pointed out here that the degree of inbreeding only influences the immediacy of the effects and, except that a high inbreeding coefficient will reduce the ultimate consequences through giving less time for heterozygous effects, will affect only slightly the total number of casualties. Similarly, the degree of heterozygous effect cannot change this number by more than a factor of two, and in no way can it be better to have, than not to have, a heterozygous effect.

If there were no selective disadvantage of the heterozygote, the simplest way ${ }^{34}$ of treating an extra load of recessives would be to assume a balance between the birth and death of mutant alleles. In a dominant, each allele takes one individual to a genetic death, in a recessive a pair of alleles are required so that each recessive leads on average to half a death. Whether these deaths necessarily follow in an affected genotype, or are the cumulative result of impaired viability, does not affect the argument. In dominants, the incidence would decline at a geometrical rate, while in recessives most of the casualties would be exported to the distant future. The inbreeding rate affects the half life of the mutations, but not their eventual capacity to induce death. In the absence of inbreeding (complete miscegenation), the half life of some recessives might well exceed the life expectation of a species. Thus, if 1000 recessive lethals were randomly distributed over $10^{9}$ individuals mating at random, the proportion surviving per generation would be 0.999999 , giving a half life of over a million years in man.

\section{Discussion}

These estimates suggest that risks of recessive disease to children from an extra $1 \mathrm{cGy}(1 \mathrm{rad}) \mathrm{X}$ or $\gamma$ radiation to parents in a population of one million would amount to less than one affected subject in the first generation after exposure and about two in the fourth (when induced recessives can become homozygous), with a total of about 10 in the first 10 generations. However, these figures depend greatly on the extent of inbreeding within the population and, to a lesser extent, on the level of selective disadvantage fixed for recessive heterozygotes. Effects of the chance spread or extinction of induced mutant alleles should also be recognised; these will tend to reduce the number of different mutants in an exposed population with succeeding generations, but not the total number of mutant heterozygotes.

The only other recent attempt to quantify recessive risks is that of Oftedal, ${ }^{35}$ but his figures (made on the assumption that recessive mutant alleles are induced with an efficiency $7 \cdot 2$ times that for dominants and that the number of genes that can mutate in the two categories is effectively the same) refer to induced recessive mutations rather than to actual cases of recessive disease, so are not comparable with our own.

Although the number of persons who would suffer the homozygous effects of induced recessives in the generations following radiation exposure would be markedly lower than for the heterozygous effects of induced dominants, yet it should be remembered that the actual detriment from most human recessives tends to be more severe than for dominants and they also tend to be manifest earlier. ${ }^{6}$ Such factors should be taken into account in any definitive assessment.

The magnitude of the risks from mutational disease after radiation exposure will depend partly on whether the dominants and recessives concerned are mutationally maintained, or have a high frequency because of heterozygous advantage. As already pointed out, it seems probable that certain recessive diseases, such as cystic fibrosis and the haemoglobinopathies, are or have been so main- 
tained. This does not mean, however, that recessive risks are lower than have been calculated because (1) for the risk of interaction of induced recessives with pre-existing recessives the important factor is the actual gene frequency of deleterious recessives in the population, which is increased if certain conditions show heterozygote advantage; (2) it seems very unlikely that radiation induced recessives would show heterozygote advantage, since the alleles involved seem to be mainly associated with complete absence of function in the gene concerned and often in neighbouring genes as well; and (3) the environment necessary for heterozygote advantage to be manifest might disappear, as in the case of the sickling trait in many countries. For dominants, however, where the radiation doubling dose is applied to the overall frequency in order to determine the number of extra cases of disease, frequencies of conditions likely to be maintained by, or to have had, heterozygous advantage should be excluded. Perhaps also should those conditions, like retinoblastoma and some other heritable cancers, which seem to be recessives (sometimes deficiencies) at the tissue level, in which somatic mutation, somatic recombination, or other phenomena are acting on the heterozygote to give a quasi-dominant condition. ${ }^{36}{ }^{37}$ Taken together, this group of dominant and quasi-dominant diseases has a joint incidence of at least $10^{-3}$.

We have assumed a $2 \cdot 5 \%$ heterozygote disadvantage for induced recessives, but the extent to which heterozygosity per se will lead to detrimental effects, and what the nature of these are likely to be, is still very uncertain. In previous risk estimates, ${ }^{4} 6$ it has been assumed that any heterozygous effects of recessives will be included in the dominant category. It seems much more likely, however, that they will be manifest as a slight increase in susceptibility to disease or to malignancy, or a slight decrease in intellect, sense organs, or physique. ${ }^{138}$ If so, they will have been excluded from recent risk estimates but should not be ignored in future ones.

The authors thank Dr Mary F Lyon FRS for her critical review of an earlier draft. Earlier versions also benefited from being discussed at genetic sessions of UNSCEAR, especially from the suggestions of Professors P S Harper and W J Schull.

\footnotetext{
References

1 Muller HJ. Our load of mutations. Am J Hum Genet 1950:2:111-76.

2 UNSCEAR. Ionizing radiation: levels and effects. Vol 2. Effects. New York: United Nations, 1972.

${ }^{3}$ BEIR. The effects on populations of exposure to low levels of ionizing radiation. Washington: National Academy of Sciences, National Research Council, 1972.
}

${ }^{4}$ BEIR. The effects on populations of exposure to low levels of ionizing radiation. Washington: National Academy of Sciences, National Research Council, 1980.

5 UNSCEAR. Sources and effects of ionizing radiation. New York: United Nations, 1977.

${ }^{6}$ UNSCEAR. Ionizing radiation: sources and biological effects. New York: United Nations, 1982.

7 Oftedal P, Searle AG. An overall genetic risk assessment for radiological protection purposes. J Med Genet 1980;17:15-20.

${ }^{8}$ UNSCEAR. Report of the United Nations Scientific Committee on the Effects of Atomic Radiation. New York: United Nations, 1966.

y Carter CO. Contribution of gene mutations to genetic disease in humans. Prog Mutat Res 1983;3:1-8.

9a Kacser H, Burns JA. The molecular basis of dominance. Genetics 1981;97:639-66.

${ }^{10}$ Gedde-Dahl T. Epidermolysis bullosa. Oslo: Universitetsforlaget, 1970.

1 McKusick VA. Mendelian inheritance in man. 6th ed. Baltimore: Johns Hopkins University Press, 1983.

12 Peters J. Mouse gene list. Mouse News Lett 1984;70:6-44.

${ }_{13}$ Harper PS. Myotonic dystrophy. Philadelphia: Saunders, 1979.

14 Reed TE, Chandler JH, Hughes EM, Davidson RT. Huntington's chorea in Michigan: demography and genetics. Am J Hum Genet 1958;10:201-25.

15 Shaw M, Caro A. The mutation rate to Huntington's chorea. $J$ Med Genet 1982;19:161-7.

16 Carter CO. Monogenic disorders. J Med Genet 1977;14:316-20.

17 Russell LB. Definition of functional units in a small chromosomal segment of the mouse and its use in interpreting the nature of radiation-induced mutations. Mutat Res 1971;11: $107-23$.

${ }^{18}$ Lyon MF, Papworth DG, Phillips RJS. Dose-rate and mutation frequency after irradiation of mouse spermatogonia. Nature 1972;238:101-4.

19 Phillips RJS. Comparison of mutation induced by acute $\mathrm{X}$ and chronic gamma irradiation in mice. Br J Radiol 1961;34:261-4.

20) Russell WL, Russell LB, Kelly EM. Radiation dose rate and mutation frequency. Science 1958;128:1546-50.

21 Lyon MF, Morris T. Gene and chromosome mutation after large fractionated radiation doses to mouse spermatogonia. Mutat Res 1969;8:191-8.

22 Searle AG, Beechey CV. A specific locus experiment with mainly dominant visible results. Genet Res 1985:45:224A.

${ }^{23}$ Russell WL, Kelly EM. Mutation frequencies in male mice and the estimation of genetic hazards of radiation in man. Proc Natl Acad Sci USA 1982;79:542-4.

${ }^{24}$ Russell WL. Mutation frequencies in female mice and the estimation of radiation hazards in women. Proc Natl Acad Sci USA 1977;74:3523-7.

25. Searle AG. Mutation induction in mice. Adv Radiat Biol 1974:4:131-207.

${ }^{26}$ Lüning KG. Searle AG. Estimates of genetic risks from ionizing irradiation. Mutat Res 1971:12:291-304.

27 Cavalli-Sforza LL, Bodmer WF. The genetics of human populations. San Francisco: Freeman. 1971.

2* Morton NE. Crow JF, Muller HJ. An estimate of the mutational damage in man from data on consanguineous marriages. Proc Natl Acad Sci USA 1956:42:855-63.

29 Edwards JH. The mutation rate in man. Prog Med Genet 1974:10:1-16.

3) Morton NE. Mutation rates for human autosomal recessives. In: Hook EB, Porter IH, eds. Population and biological aspects of human mutation. New York: Academic Press, 1981:65-89.

3 Schull WJ. Otake M. Neel JV. Genetic effects of the atomic bombs: a reappraisal. Science 1981;213:1220-7.

32 Lüning KG. Dominant effects of recessive lethals in mice. II. Viability and mating ability. Mutat Res 1969:8:573-80.

${ }^{33}$ Crow JF. Can we assess genetic risks? In: Radiation research. 
(Proc VI Int Congr Radiat Res, Tokyo). Tokyo: Japan Association for Radiation Research, 1979:70-8.

${ }^{34}$ Haldane JBS. The causes of evolution. London: Longmans Green, 1932.

${ }^{35}$ Oftedal P. Genetic damage following nuclear war. In: Effects of nuclear war on health and health services. Geneva: WHO, 1984:163-74.

36 Cairns J. Familial cancers. Nature 1984;307:116.
${ }^{37}$ Gilbert F. Retinoblastoma and recessive alleles in tumorigenesis. Nature 1983;305:761-2.

38 Vogel F. Clinical consequences of heterozygosity for autosomalrecessive diseases. Clin Genet 1984;25:381-415.

Correspondence and requests for reprints to Dr A G Searle, MRC Radiobiology Unit, Chilton, Didcot, Oxon OX11 0RD. 